\title{
Localization and circulating currents in curved graphene devices
}

\author{
G. M. M. Wakker, Rakesh P. Tiwari, and M. Blaauboer \\ Delft University of Technology, Kavli Institute of Nanoscience, Department of Quantum Nanoscience, Lorentzweg 1, \\ 2628 CJ Delft, The Netherlands
}

(Received 26 May 2011; revised manuscript received 5 October 2011; published 7 November 2011)

\begin{abstract}
We calculate the energy spectrum and eigenstates of a graphene sheet that contains a circular deformation. Using time-independent perturbation theory with the ratio of the height and width of the deformation as the small parameter, we find that due to the curvature the wave functions for the various states acquire unique angular asymmetry. We demonstrate that the pseudomagnetic fields induced by the curvature result in circulating probability currents.
\end{abstract}

DOI: 10.1103/PhysRevB.84.195427

\section{INTRODUCTION}

Graphene, a monolayer of carbon atoms in a honeycomb lattice, has attracted a lot of interest in the last decade. ${ }^{1-3}$ Along with many interesting electronic transport properties ${ }^{4}$ graphene has some intriguing structural and mechanical properties. ${ }^{5}$ A suspended graphene sheet can be deflected by applying a gate voltage. ${ }^{6}$ Early on, curvature induced by topological defects was studied in the context of carbon nanotubes and fullerenes. ${ }^{7-10}$ Graphene sheets deposited on a substrate naturally show corrugations, ${ }^{11,12}$ which can be modeled as defects. ${ }^{13}$ Deformations in a graphene sheet can provide a mechanism for spin relaxation, which is stronger than relaxation due to intrinsic spin orbit interactions. ${ }^{14}$ These deformations can also lead to significant effective pseudomagnetic fields. ${ }^{15}$ Two recent works have investigated properties of pseudomagnetic fields in graphene sheets: In Ref. 16 de Juan et al. investigate Aharonov-Bohm interferences in the local density of states due to a fictitious strain-induced magnetic field, and in Ref. 17 Abedpour et al. calculate the pseudomagnetic field induced by shear stress in circular graphene rings.

The purpose of this article is to investigate the possibility of exploiting curvature, induced by elastic deformations, in a graphene sheet for generating states with circulating currents. We focus on systems where the curvature in the graphene sample can be controlled externally. A possible way of realizing such systems is to suspend graphene samples on a substrate where an annulus has been etched away. Modeling the curvature as a Gaussian-shaped bump and using perturbation theory, we show that it is possible to generate localized circulating carriers in the ground state for such systems.

The rest of the article is organized as follows. In Sec. II, we describe the deformations studied and provide details of the perturbation theory method used later on for calculating the probability densities and the probability density current. In Sec. III, we present the analytical and numerical results obtained using a single-valley Hamiltonian. In Sec. IV, we discuss how these results change when the valley degeneracy is taken into account. We conclude our article with a summary in Sec. V.

\section{FORMALISM}

According to the standard theory of elasticity ${ }^{13,18-20}$ the effect of curvature can be captured by including a scalar $\left(V_{1}\right)$
PACS number(s): 73.22.Pr, 61.48.Gh, 72.80.Vp

and vector $\left(V_{2}=A_{p s}^{x}-i A_{p s}^{y}\right)$ potential in the Hamiltonian ${ }^{13}$ with $V_{1}=g_{1}\left(u_{x x}+u_{y y}\right)$ and $V_{2}=g_{2}\left(u_{x x}-u_{y y}+2 i u_{x y}\right)$. Here $u_{i j}$ (with $i, j \in\{x, y\}$ ) is the usual deformation tensor,

$$
u_{i j}=\frac{1}{2}\left(\frac{\partial \bar{u}_{i}}{\partial x_{j}}+\frac{\partial \bar{u}_{j}}{\partial x_{i}}+\sum_{k \in\{x, y, z\}} \frac{\partial \bar{u}_{k}}{\partial x_{i}} \frac{\partial \bar{u}_{k}}{\partial x_{j}}\right),
$$

with $\bar{u}_{i}=\bar{u}_{i}(\mathbf{r}), i \in\{x, y, z\}$, the Cartesian components of the average displacements. The coupling constants $g_{1}$ and $g_{2}$ are known from transport measurements. ${ }^{13}$ We assume that the length scales associated with the deformation are much larger than $a_{c c}$, where $a_{c c} \sim 1.4 \AA$, represents the lattice constant of graphene, so that intervalley scattering can be neglected. The single-valley Dirac Hamiltonian for the system in the absence of external magnetic fields is given by

$$
\mathcal{H}=v_{F}\left(\begin{array}{cc}
-e V_{1} & p_{x}-i p_{y}+e V_{2} \\
p_{x}+i p_{y}+e V_{2}^{*} & -e V_{1}
\end{array}\right) .
$$

Here $v_{F}$ is the Fermi velocity and $\mathbf{p}=-i \hbar \nabla$ represents the momentum operator. Now we focus on the shape of the deformation. In particular we consider two deformations: (a) a Gaussian centered at the origin (i.e., $u_{z}(\mathbf{r})=\mathcal{A} e^{-\frac{x^{2}+y^{2}}{b^{2}}}$ ) and (b) a ring-shaped Gaussian deformation modeled as $u_{z}(\mathbf{r})=$ $\mathcal{A} e^{-\frac{\left(x-c x / \sqrt{\left.x^{2}+y^{2}\right)^{2}+\left(y-c y / \sqrt{x^{2}+y^{2}}\right)^{2}}\right.}{b^{2}}}=\mathcal{A} e^{-\frac{\left(\sqrt{x^{2}+y^{2}}-c\right)^{2}}{b^{2}}}$. These deformations are schematically depicted in Figs. 1(a) and 1(b). We consider deformations with small height and large width and apply perturbation theory, using $\alpha \equiv \mathcal{A} / b$ as the small parameter. In polar coordinates the potentials $V_{1}$ and $V_{2}$ are given by $V_{1}=2 g_{1} \frac{\mathcal{A}^{2}}{b^{2}} f(r)$ and $V_{2}=2 g_{2} \frac{\mathcal{A}^{2}}{b^{2}} e^{i 2 \theta} f(r)$, where

$$
f(r)= \begin{cases}\frac{r^{2}}{b^{2}} e^{-2 r^{2} / b^{2}} & \text { for type (a) deformations } \\ \frac{(r-c)^{2}}{b^{2}} e^{-2(r-c)^{2} / b^{2}} & \text { for type (b) deformations. }\end{cases}
$$

The Hamiltonian Eq. (2) can be written in polar coordinates as $\mathcal{H}=\mathcal{H}_{0}+\mathcal{H}_{1}$ with

$$
\mathcal{H}_{0}=-i \hbar v_{F}\left(\begin{array}{cc}
0 & e^{-i \theta}\left(\partial_{r}-\frac{i}{r} \partial_{\theta}\right) \\
e^{i \theta}\left(\partial_{r}+\frac{i}{r} \partial_{\theta}\right) & 0
\end{array}\right),
$$

and

$$
\mathcal{H}_{1}=2 e \alpha^{2} f(r)\left(\begin{array}{cc}
-g_{1} & g_{2} e^{2 i \theta} \\
g_{2} e^{-2 i \theta} & -g_{1}
\end{array}\right)
$$


(a)

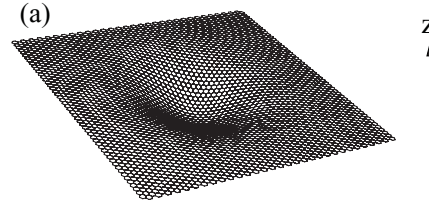

(b)

(d)

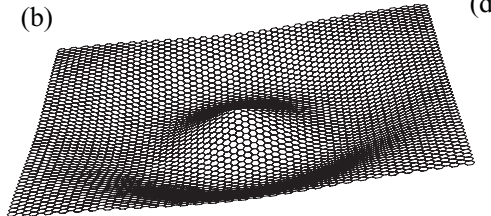

FIG. 1. (Color online) (a) Schematic of type (a) deformation. (b) Schematic of type (b) deformation. (c) Pseudomagnetic field produced by type (a) deformation. (d) Pseudomagnetic field produced by type (b) deformation. See the text for further details.

Here $\mathcal{H}_{0}$ describes the unperturbed system, which is a flat circular graphene sheet of radius $R$ and $\mathcal{H}_{1}$ is the perturbation. The normalized eigenstates of the unperturbed Hamiltonian $\mathcal{H}_{0}$ are given by ${ }^{21}$

$$
\Psi_{k, m}(r, \theta)=\sqrt{\frac{1}{2}}\left(\begin{array}{c}
J_{m}(r k) e^{i m \theta} \\
i s J_{m+1}(r k) e^{i(m+1) \theta}
\end{array}\right),
$$

where $J_{m}(r k)$ is the Bessel function of integer order $m$ with energies $\epsilon_{m, k}=s \hbar v_{F} k . s= \pm 1$ represents the conduction and the valence band. It should be noted that the states described by Eq. (6) are also eigenfunctions of the $z$ component of the total angular momentum $\hat{L}_{z}$ :

$$
\hat{L}_{z} \Psi_{k, m}=\left(-i \hbar \partial_{\phi}+\frac{\hbar}{2} \hat{\sigma}_{z}\right) \Psi_{k, m}=\left(m+\frac{1}{2}\right) \Psi_{k, m} .
$$

Now we impose infinite mass boundary conditions, ${ }^{22}$ which are valid in the regime $R \gg a_{c c}$. This can be done individually for each $m$ and results in the eigenvalue equation

$$
J_{m}\left(k_{m, l} R\right)=J_{m+1}\left(k_{m, l} R\right),
$$

where the integer $l$ represents the ascending order of the values $\left|k_{m, l}\right|$ that satisfy the above equation. The eigenvalues for $m$ and $-m-1$, which correspond to equal and opposite total angular momenta, are not degenerate. The spectrum resulting from Eq. (8) is discrete due to the finiteness of the graphene sheet, and exhibits a band gap at the Dirac point $\left(k_{m, l}=0\right)$. The full spectrum is given in Refs. 22,23. The solution of Eq. (8), closest to the Dirac point is given by $k_{-1,-1}=-\frac{1.4347}{R}$ and $k_{0,1}=\frac{1.4347}{R}$. The magnitude of the band gap is thus $2 \hbar v_{F} \frac{1.4347}{R} \approx 30 \mathrm{meV}$ for $R=50 \mathrm{~nm} .^{24}$ It is useful to write the perturbation Hamiltonian $\mathcal{H}_{1}$ as

$$
\mathcal{H}_{1}=\frac{2 e \alpha^{2}}{\beta^{2}} f(x)\left(\begin{array}{cc}
-g_{1} & g_{2} e^{2 i \theta} \\
g_{2} e^{-2 i \theta} & -g_{1}
\end{array}\right),
$$

where $\beta \equiv b / R$ and $x=r / R$. The form of the deformation is now given as

$\beta^{2} f(x)= \begin{cases}x^{2} e^{-2 \frac{x^{2}}{\beta^{2}}} & \text { for type (a) deformations } \\ (x-\gamma)^{2} e^{-2 \frac{(x-\gamma)^{2}}{\beta^{2}}} & \text { for type (b) deformations. }\end{cases}$
Here $\gamma \equiv c / R$ is a measure of the location of the deformation for type (b) deformations. The vector potential in our formalism generates a pseudomagnetic field $\mathbf{B}_{\mathrm{ps}}=\nabla \times \mathbf{A}_{\mathrm{ps}}$, perpendicular to the graphene sheet. For type (a) deformations this magnetic field is

$$
\mathbf{B}_{\mathrm{ps}}^{(a)}=8 g_{2} \alpha^{2} \frac{r^{3}}{b^{4}} e^{-2 \frac{r^{2}}{b^{2}}} \sin (3 \theta) \hat{z},
$$

as sketched in Fig. 1(c). Similarly, for type (b) deformations

$$
\begin{aligned}
\mathbf{B}_{\mathrm{ps}}^{(b)}= & \frac{4 g_{2} \alpha^{2}}{b^{4} r} e^{-2 \frac{(r-c)^{2}}{b^{2}}}\left(6 c^{2} r^{2}+2 r^{4}-2 c^{3} r-6 c r^{3}\right. \\
& \left.+b^{2} c^{2}-b^{2} c r\right) \sin (3 \theta) \hat{z},
\end{aligned}
$$

as shown in Fig. 1(d). Note that the pseudomagnetic fields Eqs. (11) and (12) are proportional to $\alpha^{2}$, and for very sharp deformations can be significant in magnitude. These pseudomagnetic fields are not real magnetic fields but a consequence of the deformation. Although these fields are zero when averaged over the entire circular graphene sheet [as can also be seen from Figs. 1(c) and 1(d)], they can locally be quite large (see the discussion at the end of the article).

\section{RESULTS}

Now we calculate the corrections due to the curvature to the energy spectrum and eigenfunctions up to second order in $\alpha^{2}$. The energy eigenvalues are then given by $\epsilon_{k_{m, l}}=\epsilon_{k_{m, l}}^{(0)}+\epsilon_{k_{m, l}}^{(1)}+$ $\epsilon_{k_{m, l}}^{(2)}$. Similarly, the wave functions are given by $\Psi_{k_{m, l}}=\Psi_{k_{m, l}}^{(0)}+$ $\Psi_{k_{m, l}}^{(1)}$. The unperturbed eigenvalues $\epsilon_{k_{m, l}}^{(0)}$ and wave functions $\Psi_{k_{m, l}}^{(0)}$ are given by $\epsilon_{k_{m, l}}^{(0)}=s \hbar v_{F} k_{m, l}$ and

$$
\Psi_{k_{m, l}}^{(0)}=A_{m l}\left(\begin{array}{c}
J_{m}\left(r k_{m, l}\right) e^{i m \theta} \\
i s J_{m+1}\left(r k_{m, l}\right) e^{i(m+1) \theta}
\end{array}\right),
$$

where $A_{m l}$ is the normalization factor given by

$$
A_{m l}=2 \int_{0}^{1}\left[J_{m}^{2}\left(k_{m, l} x\right)+J_{m+1}^{2}\left(k_{m, l} x\right)\right] x d x .
$$

The corrections $\epsilon_{k_{m, l}}^{(1)}, \epsilon_{k_{m, l}}^{(2)}$, and $\Psi_{k_{m, l}}^{(1)}$ are calculated by standard time-independent nondegenerate perturbation theory ${ }^{25}$ and we obtain

$$
\begin{aligned}
\epsilon_{k_{m} l}^{(1)} & =-\frac{4 e \alpha^{2} g_{1}}{\beta^{2}} \frac{\int_{0}^{1} f(x)\left[J_{m}^{2}\left(k_{m, l} x\right)+J_{m+1}^{2}\left(k_{m, l} x\right)\right] x d x}{A_{m l}^{2}} \\
\epsilon_{k_{m, l}}^{(2)} & =\left(2 e \alpha^{2}\right)^{2} \sum_{k_{m^{\prime} l^{\prime}} \neq k_{m, l}} \frac{\left|\mathcal{V}_{k_{m^{\prime} l^{\prime}}, k_{m, l}}\right|^{2}}{\epsilon_{k_{m, l}}^{0}-\epsilon_{k_{m^{\prime} l^{\prime}}}^{0}} \\
\Psi_{k_{m, l}}^{(1)} & =2 e \alpha^{2} \sum_{k_{m^{\prime}, l^{\prime}} \neq k_{m, l}} \frac{\mathcal{V}_{k_{m^{\prime}, l^{\prime}} k_{m, l}}}{\epsilon_{k_{m, l}}^{0}-\epsilon_{k_{m^{\prime}, l^{\prime}}}^{0}} \Psi_{k_{m^{\prime}, l^{\prime}}}^{(0)} .
\end{aligned}
$$

Here the matrix element $\mathcal{V}_{k_{m^{\prime}, l^{\prime}} ; k_{m, l}}$ is given by

$$
\begin{aligned}
\mathcal{V}_{k_{m^{\prime}, l^{\prime}} k_{m, l}}= & \frac{1}{A_{m^{\prime} l^{\prime}} A_{m l} \pi \beta^{2}} \int_{0}^{2 \pi} \int_{0}^{1} e^{i\left(m-m^{\prime}\right) \theta} f(x) \\
& \times\left[J_{m}\left(k_{m, l} x\right),-i s J_{m+1}\left(k_{m, l} x\right) e^{-i \theta}\right] \\
& \times\left[\begin{array}{cc}
-g_{1} & g_{2} e^{2 i \theta} \\
g_{2} e^{-2 i \theta} & -g_{1}
\end{array}\right]\left[\begin{array}{c}
J_{m^{\prime}}\left(k_{m^{\prime}, l^{\prime}} x\right) \\
i s J_{m^{\prime}+1}\left(k_{m^{\prime}, l^{\prime}} x\right) e^{i \theta}
\end{array}\right] x d x d \theta .
\end{aligned}
$$



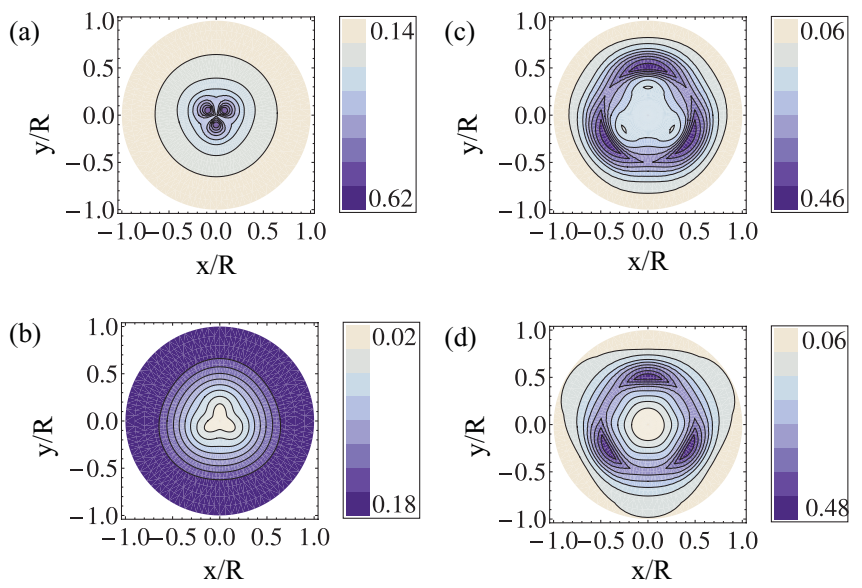

FIG. 2. (Color online) Contour plot of $\Psi_{k_{m, l}}^{\dagger} \Psi_{k_{m, l}}$ (calculated using a single-valley Hamiltonian) (a) for the lowest electronic state of type (a) deformation, (b) for the first excited state of type (a) deformation, (c) for the lowest electronic state of type (b) deformation and (d) for the first excited state of type (b) deformation. Parameters used are $\alpha=0.04, \beta=0.1, \gamma=0.4, e g_{1}=3 \mathrm{eV}, e g_{2}=2.276 \mathrm{eV}^{13}$. See the text for further details.

The angular integral in Eq. (16) is only nonzero if $m=m^{\prime}, m=$ $m^{\prime}+3$, or $m=m^{\prime}-3$, which simplifies the calculations.

Now we present our numerical results. In principle the sum in the expressions for $\epsilon_{k_{m, l}}^{(2)}$ and $\Psi_{k_{m, l}}^{(1)}$ [Eq. (15)] runs over an infinite number of values of $k_{m, l}$, but we restrict the sum to values of $k_{m, l}$ such that $m \in(-6,5)$ and $l \in(-15,15)$. We have verified that the results obtained by using this restricted sum presented for the lowest two electronlike and lowest two holelike eigenvalues and eigenfunctions are converged up to at least four significant digits.

In Fig. 2 we plot the probability density $\Psi_{k_{m, l}}^{\dagger} \Psi_{k_{m, l}}$ over the entire graphene sheet for the lowest electronic state (i.e., $k_{m, l}=$ $1.4347 / R$ ) and the first excited electronic state (i.e., $k_{m, l}=$ $2.62987 / R$ ) for both type (a) and (b) deformations within a single valley. Below we discuss these single valley results in detail. A contour plot of $\Psi_{k_{m, l}}^{\dagger} \Psi_{k_{m, l}}$ is shown in Fig. 2(a) for the lowest electronic state (ground state) for a central Gaussian [type (a)] deformation. We see that there is a unique angular dependence of the probability density. Similarly, Fig. 2(b) shows that $\Psi_{k_{m, l}}^{\dagger} \Psi_{k_{m, l}}$ for the first excited electronic state of a type (a) deformation is also angle dependent. These angular dependencies are in agreement with the pseudomagnetic field $\mathbf{B}_{\mathrm{ps}}^{(a)}$ generated by such a deformation. For a ring-shaped Gaussian it is also evident from Figs. 2(c) and 2(d) that the probability densities $\Psi_{k_{m, l}}^{\dagger} \Psi_{k_{m, l}}$ are also angle dependent for both the ground state and the first excited state for type (b) deformations. It should be noted that the probability density is high around the location of the deformation $(r=0.4 R)$. As we increase $\alpha$, the effect of deformation-induced localization of the wave functions for both types of deformations increases. The angle dependence of the probability densities comes primarily from the vector potential $\left(A_{\mathrm{ps}}\right)$, the main effect of the scalar potential is to shift the energy eigenvalues Eq. (15).

A localized probability density results in circulating currents, which in turn generate local magnetic fields. In order to investigate this effect we calculate the probability current

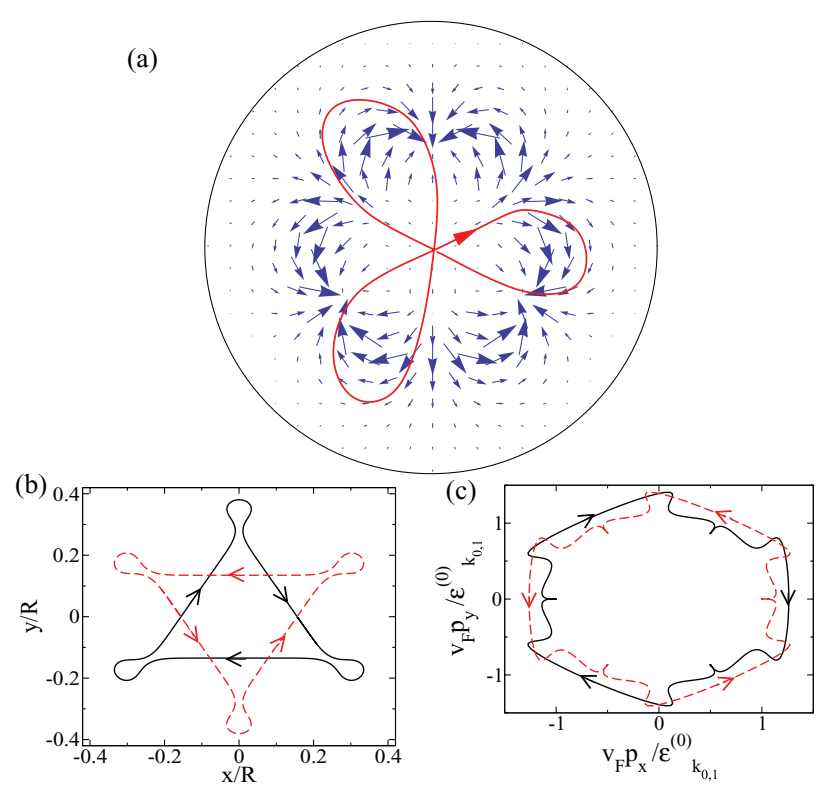

FIG. 3. (Color online) (a) Vector field plot of $\mathbf{j}$ over the entire graphene sheet for the lowest electronic state for a ring-shaped Gaussian [type (b)] deformation (calculated using a single-valley Hamiltonian). (b) Classical trajectory in real space calculated using Eq. (15). Solid (black) and dashed (red) lines represent two different sublattices. (c) Classical trajectory in momentum space calculated using Eq. (15). Again solid (black) and dashed (red) lines represent two different sublattices. See the text for further details.

density $\mathbf{j} \equiv v_{F} \Psi_{k_{m, l}}^{\dagger} \sigma \Psi_{k_{m, l}}$. Figure 3(a) shows a vector field plot of $\mathbf{j}$ over the entire graphene sheet for the lowest electronic state of a ring-shaped Gaussian [type (b)] deformation. The solid line is drawn just to guide the eye. Localization of the carriers is a repercussion of the topological effect of the pseudomagnetic field. To elucidate this further we plot in Fig. 3 the classical trajectories calculated from the corresponding classical Hamiltonian

$$
\mathcal{H}_{\text {class }}= \pm v_{F} \sqrt{\left(p_{x}+\frac{A_{\mathrm{ps}}^{x}}{v_{F}}\right)^{2}+\left(p_{y}+\frac{A_{\mathrm{ps}}^{y}}{v_{F}}\right)^{2}} .
$$

Figure 3(b) shows the position space cyclotron orbits for the ground state of the ring shaped Gaussian deformation. ${ }^{23}$ The two different sublattices of graphene have different orbits as is shown by the solid and the dashed lines. Figure 3(c) shows the corresponding momentum space closed orbits. These classical orbits exhibit remarkable similarity to the quantum-mechanical probability density and the probability density current shown in Figs. 2(c) and 3(a). These circulating orbits produce local magnetic fields (which are zero when averaged over the entire graphene sheet). It should be stressed that the existence of these magnetic fields does require the carriers to be valley polarized, since otherwise the degenerate carriers in the other valley will cancel these local magnetic fields exactly. As has been suggested recently, a special kind of line defect can be used in graphene as a valley filter. ${ }^{26}$ Alternately, weak intervalley scattering has been shown to lift the valley degeneracy. ${ }^{16,27}$

Experimentally one can measure the angular distribution of the probability density using a scanning tunneling microscope 
(STM). To get an estimate for the order of magnitude of the magnetic fields produced we approximate the current density depicted in Fig. 3(a) by a circular wire of radius $0.4 R$, where six equal arcs on the circumference carry alternating countercirculating constant currents. For typical carrier densities of $10^{12}$ $\mathrm{cm}^{-2}$ the resulting magnetic fields are then of order $100 \mu \mathrm{T}$. It should be stressed that experimental detection of these small real magnetic fields is quite challenging, and one possibility is using a single nitrogen-vacancy (NV) impurity in diamond. ${ }^{28}$

\section{EFFECT OF THE OTHER VALLEY}

In this section we present the results obtained using the full two-valley Hamiltonian. In the above calculations the states with wave vector $\mathbf{k}$ were in the valley centered at the corner of the Brillouin zone with wave vector $\mathbf{K}=4 \pi /\left(3 a_{c c}\right) \hat{x}$. The other independent valley of excitations is located at the opposite corner of the Brillouin zone with wave vector $\mathbf{K}^{\prime}=$ $-\mathbf{K}$. The wave function $\Psi$ now becomes a four component spinor which can be represented as $\Psi=\left(\psi_{A}, \psi_{B},-\psi_{B}^{\prime}, \psi_{A}^{\prime}\right)^{\mathrm{T}}$ and the Hamiltonian is given by ${ }^{29}$

$$
\mathcal{H}_{\text {both valleys }}(\mathbf{p})=v_{F}\left(\begin{array}{cc}
\mathbf{p} \cdot \sigma & 0 \\
0 & \mathbf{p} \cdot \sigma
\end{array}\right) \text {. }
$$

In Fig. 4 we plot the probability density $\Psi_{k_{m, l}}^{\dagger} \Psi_{k_{m, l}}$ over the entire graphene sheet for the lowest electronic state (i.e., $\left.k_{m, l}=1.4347 / R\right)$ and the first excited electronic state (i.e., $k_{m, l}=2.62987 / R$ ) for both type (a) and (b) deformations

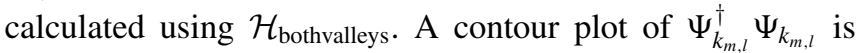
shown in Figs. 4(a) and 4(c) for the lowest electronic state (ground state) of a central Gaussian [type (a)] deformation and a ring-shaped Gaussian [type (b)] deformation respectively. Figure 4(b) is a contour plot of $\Psi_{k_{m, l}}^{\dagger} \Psi_{k_{m, l}}$ for the first excited electronic state of type (a) deformations and Fig. 4(d) shows the same for type (b) deformations. The latter shows a clear
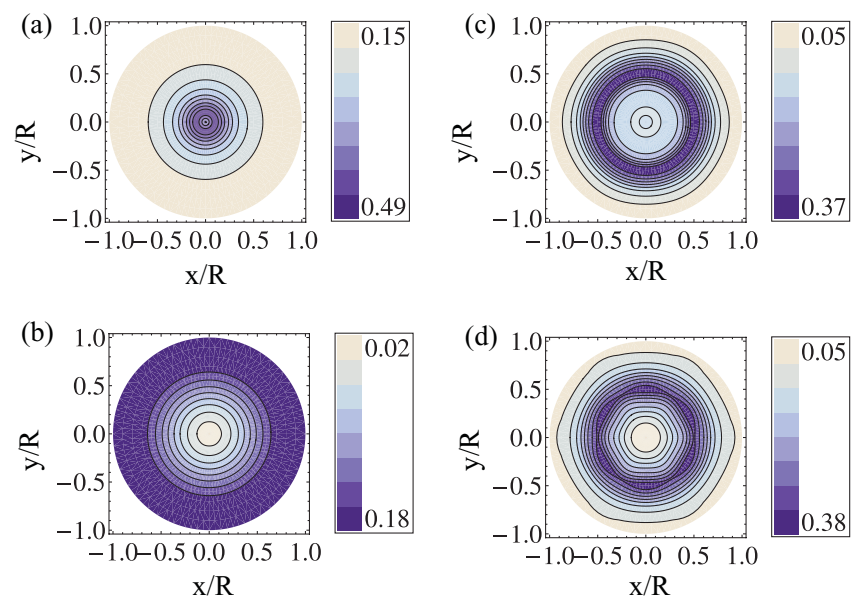

FIG. 4. (Color online) Contour plot of $\Psi_{k_{m, l}}^{\dagger} \Psi_{k_{m, l}}$ calculated using $\mathcal{H}_{\text {both valleys }}$ (a) for the lowest electronic state of type (a) deformation, (b) for the first excited state of type (a) deformation, (c) for the lowest electronic state of type (b) deformation, and (d) for the first excited state of type (b) deformation. Parameters used are $\alpha=0.04$, $\beta=0.1, \gamma=0.4, e g_{1}=3 \mathrm{eV}, e g_{2}=2.276 \mathrm{eV}^{13}$ See the text for further details. sixfold feature, whereas in the other three cases the probability density seems to be circularly symmetric. Although not visible in Figs. 4(a)-4(c), there is some angular dependence in the probability density shown in these plots too.

In order to better understand the origin of the angular dependence of the probability density we analyze Eq. (15) in some more detail. As mentioned earlier only $m=m^{\prime}, m=$ $m^{\prime}+3$, and $m=m^{\prime}-3$ terms yield a nonzero contribution to the first-order corrections of the wave function $\Psi_{k_{m, l}}^{(1)}$. For a given value of $r$ the wave function for the ground state $\Psi^{\mathrm{g}}$, can be written as $\Psi^{\mathrm{g}}=\frac{1}{\sqrt{2}}\left(\Psi_{\mathbf{K}}^{\mathrm{g}}, \Psi_{-\mathbf{K}}^{\mathrm{g}}\right)^{T}$ and similarly for the first excited state we write $\Psi^{\mathrm{e}}=\frac{1}{\sqrt{2}}\left(\Psi_{\mathbf{K}}^{\mathrm{e}}, \Psi_{-\mathbf{K}}^{\mathrm{e}}\right)^{T}$. Then we can explicitly write

$$
\begin{aligned}
\Psi_{\mathbf{K}}^{\mathrm{g}} & =\left(a_{0}^{r}+i a_{1}^{r} e^{-3 i \theta}+i a_{2}^{r} e^{3 i \theta}, i b_{0}^{r} e^{i \theta}+b_{1}^{r} e^{-2 i \theta}+b_{2}^{r} e^{4 i \theta}\right) \\
\Psi_{-\mathbf{K}}^{\mathrm{g}} & =\left(-a_{0}^{r}+i a_{1}^{r} e^{-3 i \theta}+i a_{2}^{r} e^{3 i \theta}, i b_{0}^{r} e^{i \theta}-b_{1}^{r} e^{-2 i \theta}-b_{2}^{r} e^{4 i \theta}\right) \\
\Psi_{\mathbf{K}}^{\mathrm{e}} & =\left(c_{0}^{r} e^{i \theta}+i c_{1}^{r} e^{-2 i \theta}+i c_{2}^{r} e^{4 i \theta}, i d_{0}^{r} e^{2 i \theta}+d_{1}^{r} e^{-i \theta}+d_{2}^{r} e^{5 i \theta}\right) \\
\Psi_{-\mathbf{K}}^{\mathrm{e}} & =\left(-c_{0}^{r} e^{i \theta}+i c_{1}^{r} e^{-2 i \theta}+i c_{2}^{r} e^{4 i \theta}, i d_{0}^{r} e^{2 i \theta}-d_{1}^{r} e^{-i \theta}-d_{2}^{r} e^{5 i \theta}\right),
\end{aligned}
$$

where $a_{0}^{r}, a_{1}^{r}, a_{2}^{r}, b_{0}^{r}, b_{1}^{r}, b_{2}^{r}, c_{0}^{r}, c_{1}^{r}, c_{2}^{r}, d_{0}^{r}, d_{1}^{r}$, and $d_{2}^{r}$ are real coefficients that depend on the radial coordinate $r$ and the type of the deformation. From Eq. (19) we now obtain the ground state probability density as $\left|\Psi^{\mathrm{g}}\right|^{2}=a_{0}^{r 2}+a_{1}^{r 2}+a_{2}^{r 2}+b_{0}^{r 2}+$ $b_{1}^{r 2}+b_{2}^{r 2}+2\left(a_{1}^{r} a_{2}^{r}+b_{1}^{r} b_{2}^{r}\right) \cos (6 \theta)$. Similarly the probability density of the first excited state can be written as $\left|\Psi^{\mathrm{e}}\right|^{2}=$ $c_{0}^{r 2}+c_{1}^{r 2}+c_{2}^{r 2}+d_{0}^{r 2}+d_{1}^{r 2}+d_{2}^{r 2}+2\left(c_{1}^{r} c_{2}^{r}+d_{1}^{r} d_{2}^{r}\right) \cos (6 \theta)$.

In order to highlight the angular dependence of these states, we plot in Fig. 5 the normalized probability densities $\frac{\left|\Psi^{\mathrm{g}}(r, \theta)\right|^{2}}{\left|\Psi^{\mathrm{g}}(r, \theta=0)\right|^{2}}$
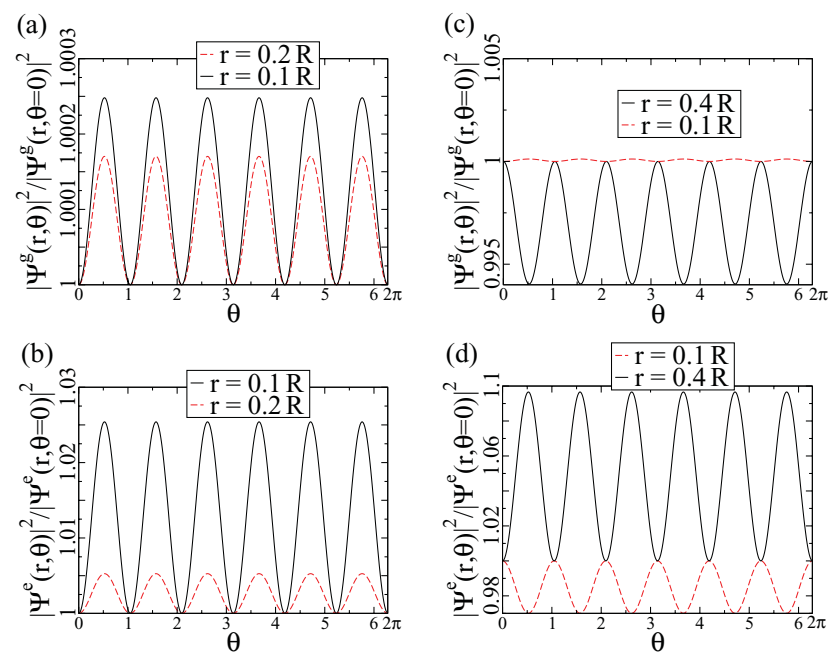

FIG. 5. (Color online) (a) $\frac{\left|\Psi^{\mathrm{g}}(r, \theta)\right|^{2}}{\left|\Psi^{\mathrm{g}}(r, \theta=0)\right|^{2}}$ for a type (a) deformation. The solid (black) line denotes $r=0.1$ and the dashed (red) line denotes $r=0.2$. (b) $\frac{\left|\Psi^{\mathrm{e}}(r, \theta)\right|^{2}}{\left|\Psi^{\mathrm{e}}(r, \theta=0)\right|^{2}}$ for a type (a) deformation. The solid (black) line denotes $r=0.1$ and the dashed (red) line denotes $r=0.2$. (c) $\frac{\left|\Psi^{\mathrm{g}}(r, \theta)\right|^{2}}{\left|\Psi^{\mathrm{g}}(r, \theta=0)\right|^{2}}$ for a type (b) deformation. The solid (black) line denotes $r=0.4$ and the dashed (red) line denotes $r=0.1$. (d) $\frac{\left|\Psi^{\mathrm{e}}(r, \theta)\right|^{2}}{\left|\Psi^{\mathrm{e}}(r, \theta=0)\right|^{2}}$ for

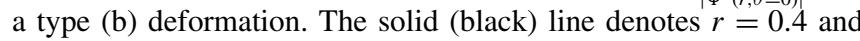
the dashed (red) line denotes $r=0.1$. Parameters used are $\alpha=0.04$, $\beta=0.1, \gamma=0.4, e g_{1}=3 \mathrm{eV}, e g_{2}=2.276 \mathrm{eV} .{ }^{13}$ See the text for further details. 
and $\frac{\left|\Psi^{\mathrm{e}}(r, \theta)\right|^{2}}{\left|\Psi^{\mathrm{e}}(r, \theta=0)\right|^{2}}$ as a function of $\theta$ for a given value of $r$. Figure 5(a) shows the angular dependence of $\frac{\left|\Psi^{\mathrm{g}}(r, \theta)\right|^{2}}{\left|\Psi^{\mathrm{g}}(r, \theta=0)\right|^{2}}$ for type (a) deformations. Figure 5(c) shows the same for type (b) deformations. Similarly, Fig. 5(b) shows $\frac{\left|\Psi^{\mathrm{e}}(r, \theta)\right|^{2}}{\left|\Psi^{\mathrm{e}}(r, \theta=0)\right|^{2}}$ for type (a) deformations and Fig. 5(d) shows the angular dependence of $\frac{\left|\Psi^{\mathrm{e}}(r, \theta)\right|^{2}}{\left|\Psi^{\mathrm{e}}(r, \theta=0)\right|^{2}}$ for type (b) deformations. It is clear from these plots that there is some angular dependence of the probability density for both the ground state and the first excited state of both types of deformations (larger for the excited states than for the ground states). Although this dependence is small, we have verified that it is not due to lack of convergence of terms in the sum of Eq. (15) and believe it to be genuinely present due to the threefold symmetry in the contribution of the angular momentum (angle $\theta$ ) to the wave function Eq. (19).

Finally, we comment on the symmetry properties of the system. The time-reversal operator $(\mathcal{T})$ for the two-valley Hamiltonian is given by $\mathcal{T}=-\left(\tau_{y} \otimes \sigma_{y}\right) \mathcal{C}$. Here $\tau_{y}$ is a $2 \times 2$ Pauli matrix acting on the valley degree of freedom, $\sigma_{y}$ is a $2 \times$ 2 Pauli matrix acting on the sublattice degree of freedom, and $\mathcal{C}$ is the complex conjugation operator. ${ }^{29}$ As mentioned above the effect of the curvature is included as a scalar potential $\left(V_{1}\right)$ and a vector potential $\left(A_{\mathrm{ps}}\right)$. So the full two-valley Hamiltonian is given by $\mathcal{H}_{\text {both valleys }}^{\text {full }}=\mathcal{H}_{\text {bothvalleys }}\left(\mathbf{p}+e \mathbf{A}_{\mathrm{ps}}\right)+e V_{1} \mathcal{I}$. Here $\mathcal{I}$ represents $4 \times 4$ identity matrix. The perturbation considered in this work does not break the time-reversal symmetry. Instead the symmetry broken by this perturbation is the socalled symplectic symmetry, ${ }^{23,29}$ represented by the operator $\mathcal{S}=i\left(\tau_{0} \otimes \sigma_{y}\right) \mathcal{C}$, where $\tau_{0}$ is a $2 \times 2$ identity matrix in the valley space. This operator $\mathcal{S}$ acts like a time-reversal operator in a single valley.

\section{CONCLUSION}

To summarize, we have calculated the energy spectrum and wave functions for two circular Gaussian shaped deformations in a graphene sheet using perturbation theory. Due to the curvature in the graphene sheet the carriers become localized around the deformation and follow circulating orbits. These circulating carriers produce local magnetic fields, which are zero when averaged over the entire sample. Interesting angular dependence of the probability density is predicted for Gaussian deformations. We suggest different ways to experimentally verify our predictions with current technologies, which will provide further insight.

\section{ACKNOWLEDGMENTS}

We thank Ya. M. Blanter, F. Konschelle, and G. Steele for stimulating discussions. This research was supported by the Dutch Science Foundation NWO/FOM.
${ }^{1}$ K. S. Novoselov, A. K. Geim, S. V. Morozov, D. Jiang, Y. Zhang, S. V. Dubonus, I. V. Grigorieva, and A. A. Firsov, Science 306, 666 (2004).

${ }^{2}$ K. S. Novoselov, D. Jiang, F. Schedin, T. J. Booth, V. V. Khotkevich, S. V. Morozov, and A. K. Geim, Proc. Natl. Acad. Sci. USA 102, 10451 (2005).

${ }^{3}$ A. H. Castro Neto, F. Guinea, N. M. R. Peres, K. S. Novoselov, and A. K. Geim, Rev. Mod. Phys. 81, 109 (2009).

${ }^{4}$ D. S. L. Abergela, V. Apalkovb, J. Berashevicha, K. Zieglerc, and T. Chakraborty, Adv. Phys. 59, 261 (2010).

${ }^{5}$ M. A. H. Vozmediano, M. I. Katsnelson, and F. Guinea, Phys. Rep. 493, 109 (2010).

${ }^{6}$ M. M. Fogler, F. Guinea, and M. I. Katsnelson, Phys. Rev. Lett. 101, 226804 (2008).

${ }^{7}$ J. González, F. Guinea, and M. A. H. Vozmediano, Phys. Rev. Lett. 69, 172 (1992).

${ }^{8}$ J. González, F. Guinea, and M. A. H. Vozmediano, Nucl. Phys. B 406, 771 (1993).

${ }^{9}$ R. Tamura and M. Tsukada, Phys. Rev. B 49, 7697 (1994).

${ }^{10}$ J. C. Charlier and G. M. Rignanese, Phys. Rev. Lett. 86, 5970 (2001).

${ }^{11}$ J. C. Meyer, A. K. Geim, M. I. Katnelson, K. S. Novoselov, T. J. Booth, and S. Roth, Nature (London) 446, 60 (2007).

${ }^{12}$ D. Garcia-Sanchez, A. M. van der Zande, A. S. Paulo, B. Lassagne, P. L. McEuen, and A. Bachtold, Nano Lett. 8, 1399 (2008).

${ }^{13}$ M. Gibertini, A. Tomadin, M. Polini, A. Fasolino, and M. I. Katsnelson, Phys. Rev. B 81, 125437 (2010).

${ }^{14}$ D. Huertas-Hernando, F. Guinea, and A. Brataas, Phys. Rev. Lett. 103, 146801 (2009).
${ }^{15}$ N. Levy, S. A. Burke, K. L. Meaker, M. Panlasigui, A. Zettl, F. Guinea, A. H. Castro Neto, and M. F. Crommie, Science 329, 544 (2010).

${ }^{16}$ F. de Juan, A. Cortijo, M. A. H. Vozmediano, and A. Cano, Nature Phys. 7, 810 (2011).

${ }^{17}$ N. Abedpour, R. Asgari, and F. Guinea, Phys. Rev. B 84, 115437 (2011).

${ }^{18}$ H. Suzuura and T. Ando, Phys. Rev. B 65, 235412 (2002).

${ }^{19}$ F. de Juan, A. Cortijo, and M. A. H. Vozmediano, Phys. Rev. B 76, 165409 (2007).

${ }^{20}$ J. L. Mañes, Phys. Rev. B 76, 045430 (2007).

${ }^{21}$ N. M. R. Peres, J. N. B. Rodrigues, T. Stauber, and J. M. B. Lopes dos Santos, J. Phys. Condens. Matter 21, 344202 (2009).

${ }^{22}$ M. V. Berry and R. J. Mondragon, Proc. R. Soc. London A 412, 53 (1987).

${ }^{23}$ K. J. Kim, Ya. M. Blanter, and K. H. Ahn, Phys. Rev. B 84, 081401 (2011).

${ }^{24}$ There already exist many proposals to open and control band gaps in graphene at the Dirac point (e.g., by cutting a nanoribbon) [see L. Brey and H. A. Fertig, Phys. Rev. B 73, 235411 (2006) for more details] and gaps induced by specific choices of substrate. [see C. Enderlein, Y. S. Kim, A. Bostwick, E. Rotenberg, and K. Horn, New J. Phys. 12, 033014 (2010)].

${ }^{25}$ J. J. Sakurai, Modern Quantum Mechanics (Pearson Education, Singapore, 1994).

${ }^{26}$ D. Gunlycke and C. T. White, Phys. Rev. Lett. 106, 136806 (2011).

${ }^{27}$ A. Chaves, L. Covaci, Kh. Yu. Rakhimov, G. A. Farias, and F. M. Peeters, Phys. Rev. B 82, 205430 (2010). 
${ }^{28}$ J. R. Maze, P. L. Stanwix, J. S. Hodges, S. Hong, J. M. Taylor, P. Cappellaro, L. Jiang, M. V. G. Dutt, E. Togan, A. S. Zibrov, A. Yacoby, R. L. Walsworth, and M. D. Lukin, Nature (London) 455, 644 (2008); G. Balasubramanian, I. Y. Chan, R. Kolesov, M. Al-Hmoud, J. Tisler, C. Shin, C. Kim, A. Wojcik, P. R. Hemmer, A. Krueger, T. Hanke, A. Leitenstorfer, R. Bratschitsch, F. Jelezko, and J. Wrachtrup, ibid. 455, 648 (2008); P. C. Maurer, J. R. Maze, P. L. Stanwix, L. Jiang, A. V. Gorshkov, A. A. Zibrov, B. Harke, J. S. Hodges, A. S. Zibrov, A. Yacoby, D. Twitchen, S. W. Hell, R. L. Walsworth, and M. D. Lukin, Nature Phys. 6, 912 (2010).

${ }^{29}$ C. W. J. Beenakker, Rev. Mod. Phys. 80, 1337 (2008). 